\title{
Impulsive Noise removal Image Enhancement Technique
}

\author{
Subrajeet Mohapatra', Pankaj Kumar Sa², Banshidhar Majhi ${ }^{3}$ \\ ${ }^{1,2.3}$ National Institute of Technology Rourkela, \\ Rourkela 769 008, Orissa, India \\ E-mail : Subrajeets@gmail compankajsa@nitrkl.ac.in bmajhi@nitrkl.ac.in
}

\section{Abstract}

Several areas like remote sensing, biomedical analysis, and computer vision require good image contrast and details for better interpretations and diagnosis's. In the literature various image enhancement algorithms have been proposed to improve the perceptual aspects of the image for poorly contrasted images. The perceptual appearance of an image may be significantly improved by modifying the high frequency components to have better edge and detail information in the image. The proposed scheme is a modification of simple unsharp mask image enhancement technique. We propose a simple method to improve the pitfalls of the existing unsharp mask technique. The idea is to remove impulsive noise while preserving the edges and to have better contrast for visual perception. Comparative analysis on standard images at different noise conditions shows that the proposed scheme, in general, outperforms the existing schemes with better results.

Key words : Image enhancement, contrast enhancement, unsharp masking, impulsive noise removal

\section{INTRODUCTION}

The purpose of image contrast enhancement is to increase the visibility of images. A good number of methods are available in the literature for enhancing different properties or components of images [1].Most of the contrast enhancement techniques can be classified into two major categories: intensity based techniques and feature based techniques. Intensity based techniques can be modeled by the form

$$
l^{\prime}(x, y)=f(I(x, y))
$$

Where, $I(X, Y)$ is the original image, $I^{\prime}(X, Y)$ is the output image after enhancement, and $f$ is the applied transformation function. This transformation is applied to the whole image that means pixels with the same gray level at different image locations of the original image are still kept the same in the enhanced image. Contrast stretching [1], histogram equalization [2] are the popular members within this category. Histogram equalization is a widely accepted image enhancement technique. Variation of this algorithm like Bihistogram Equalization [3], Multipeak histogram equalization [4], Adaptive histogram equalization is also available in the literature. Contrast Limited Adaptive histogram equalization (CLAHE) [5] is the widely accepted version of histogram equalization which overcomes the pitfalls of general histogram equalization. The basic idea behind intensity based method is a gray level transformation applied to the whole image, so that the image appears visually more distinct. The basic idea of feature based image enhancement technique is to enhance the small-scale components, which represent some specified features, of an image. Feature based methods can be represented by the form

$$
I^{\prime}(x, y)-L(x, y)+G(x, y) H_{1}(x, y) .
$$

where $L_{1}$ represents the low frequency components hich keep the basic appearance of the original image, $\mathrm{H}_{1}$ represents the high frequency components which contains the edges and detailed regions of the image and $G$ is the enhancement gain. Feature based contrast enhancement can be done in spatial domain with the help of unsharp masks. Various other methods like statistical methods $[6,7]$ have also been adopted for achieving image enhancement using feature based method. The classic linear unsharp masking (UM)is an important scheme in the feature based image enhancement category. In the UM technique, a high pass filtered scaled version of the original input image is added to itself as shown in Fig.1.Here the idea is to give more emphasis on the high frequency components such as to enhance the edges and detail information. of an image. Because of its simplicity unsharp masking is used in most of the image editing software's. Although this method works well in many applications but it suffers a major drawback which limitsits performance in certain applications. Since without removing the noise we are using a scaled version of the high pass filtered image. There is every possibility that the noise is also amplified along with the edge and detail features of the image. Since the objective of the image enhancement are to remove impulsive noise and to enhance edges and detail features of the input image. So the main objective of our method is to separate the noisy and noise-free pixels based on certain threshold value. Hence we can remove the impulsive noise and then apply synergistically the simple unsharp masking technique along with Contrast limited AHE(CLAHE) [5] for edge (1) (2) and detail features enhancement for better human perception. Here we have used a CV based adaptive threshold technique for impulsive noise detection. The 
main idea is to train the functional link artificial neural network (FLANN)[12] using a single statistical parameter coefficient of variance (CV) as input.

In Section II. the impulsive noise model along with the proposed scheme is Presented. Threshold selection using CV is discussed in Section III. Section IV. presents the results and Section $V$. gives the concluding remark.

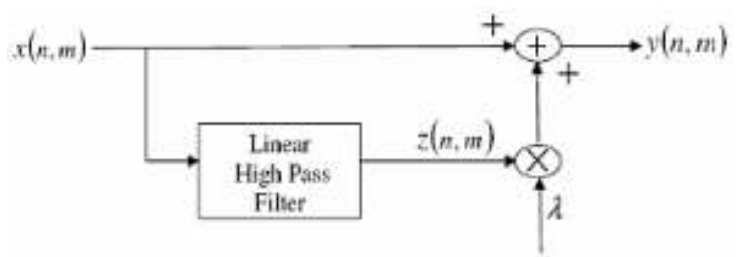

Fig. 1. Linear unsharp masking for image contrast enhancement

\section{IMPULSIVE NOISE MODEL}

Images are frequently contaminated by impulsive noise due to noisy sensors or channel transmission errors [1]. Since because of high pass filter in Unsharp Masking (UM) the scheme becomes highly sensitive to noise. There are many types of impulsive noise. Let $j X \mathrm{i}$, be the gray level of an original image $X$ at pixel location(i,j), and $\left[\mathrm{n}_{\text {max }}, n_{\min }\right.$ be the dynamic range of $X$. Let $j Y \mathrm{i}$, be the gray level of the noisy image $Y$ at pixel (i, j), and then the random valued impulsive noise may be defined as

$$
Y_{i, j}= \begin{cases}X_{i, j} & \text { with } 1-p \\ R_{i, j} & \text { with } p\end{cases}
$$

Where Ri,j $\left[n_{\max }, n_{\text {min }}\right]$ and $p$ is the noise ratio. Where as for Fixed Valued Impulsive Noise (better known as Salt and Pepper Noise) $R i, j\left\{n_{\max }, n_{\min }\right.$ max min , $\left.n R j i,\right\}$. It is usually seen that removal of Salt and Pepper Noise is easier in comparison to RVIN since behavior of RVIN pixels and its surrounding pixels are very similar. In this paper we focus only on Random Valued Impulsive Noise where $j \mathrm{Yi}$, can be of any value from min $n$ to $\max n$.So before image enhancement impulsive noise must be removed to avoid unnecessary noise amplification.

\section{PROPOSED SCHEME}

The proposed scheme consists of three phases, impulse detection, filtration and image contrast enhancement as shown in Fig. 2. Only those pixels that are classified as contaminated in the detection phase are subjected to filtration. It employs a second order difference based impulse detection mechanism at the location of the test pixel. The mathematical formulation of the scheme can be modeled as:

$$
\hat{Y}_{i, j}= \begin{cases}Y_{i, j} & d_{i, j}=1 \\ Z_{i, j} & d_{i, j}=0\end{cases}
$$

Where $Z_{i, j,}=\operatorname{median}\left\{Y_{i-k j ; k},(k, l) E W\right\}$ and $W$ is a predetermined window, usually of size $3 \times 3$ or $5 \times 5[1]$.

The filtration is performed selectively based on the decision of the impulse detector .The detection (and filtration) is done twice, once in the horizontal direction and again in the vertical direction, thus the test pixel is compared in both directions. Median filtering is also done twice immediately after each detection phase only on those pixels which are identified as contaminated so avoiding blurring of images. In the third phase we use a high pass filter for obtaining the high frequency components of the image .Followed by addition of the original image to the output of the high pass filter. This gives us an edge enhanced image without impulsive noise.

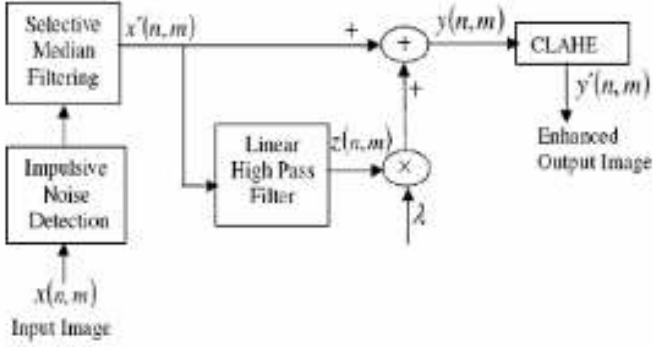

Fig. 2. Proposed model for image contrast enhancement

\section{Algorithm}

\section{Pass One}

1. Choose a test window $Y^{(T)}$ of size $3 X 3$ centered at $(i, j)$ of $Y$. Choose a sub sindow $Y^{(M)}$ of sixe $3 X 3$ centered at $(I, j)$ of $Y^{(\top)}$

2. Compute the first order $3 \times 4$ difference matrix $f d$

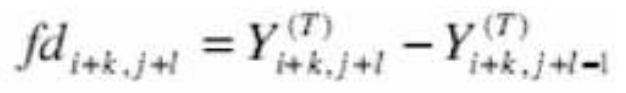

Where $r=-1,0,1$ and $s=-1,0,1,2$

3. Compute the second order $3 \times 3$ difference matrix sd fromfd

$$
s d_{i+r, j+s}=f d_{i+r, j+s+1}-f d_{i+r, j+s}
$$

Where $r=-1,0,1$ and $s=-1,0,1$ 
4. Compute the decision paramater $d$

$$
d_{i, j}= \begin{cases}0 & \text { if }\left|s d_{i, j}\right|>\theta_{1} \\ 1 & \text { otherwise }\end{cases}
$$

If $d_{i, j}$ is zero, replace the $Y_{i, j}$ pixel with the median value of its neighborhood, otherwise leave it as it is, repeat the above steps for each window from top left to bottomright corner of the noisy image.

\section{Pass two}

The window $Y^{(T)}$ is selected is of sixe $5 X 3$ centered at (i,j) of $y$ and sub window $Y^{(\mathrm{W})}$ of sixe $3 X 3$ centered at $(i, j)$ of $Y^{(T)}$ The first and second order differences are calculated in vertical fashion and the decision parameter is determined, followed by selective filtration similar to the steps described earlier. The threshold values taken here in this pass is $\theta_{2}$. The threshold values are obtained using FLANN as described in section 3. All the steps in the second iteration are repeated for each test window column wise from top-left to bottom-right corner of the image obtained from pass one to obtain the final filtered image. After impulsive noise removal is done the chance for noise amplification is reduced so now it is suitable for image enhancement using unsharp masking and CLAHE.

\section{Image Contrast Enhancement}

The filtered image output say is fed into the high pass filter to segregate the high frequency components of the image from the smooth details of the image. We can choose a suitable gain factor for amplification of image detail regions like sharp edges depending on the application where we use it. So this can be modeled in the form of

$$
y(n, m)=x^{\prime}(n, m)+\lambda z(n, m)
$$

where $z(n, m)$ is the correction signal computed as the output of a linear high pass filter. $z(n, m)$ is obtained using the relation

$$
\begin{aligned}
& z(n, m)=4 x^{\prime}(n, m)-x^{\prime}(n-1, m) \\
& -x^{\prime}(n+1, m)-x^{\prime}(n, m-1)-x^{\prime}(n, m+1)
\end{aligned}
$$

$\lambda$ is the positive scaling factor that controls the level of contrast enhancement achieved at the output. $X^{\prime}$ 's the filtered version of the original image with edges and fine details preserved .This filtered image $X$ '?s fed as input for CLAHE to obtain the final enhanced output. The proposed scheme tries to prevent amplification of impulsive noise and tries to provide better visual contrast about the image details.

\section{Adaptive threshold selection}

Artificial Neural Networks are being used in pattern classification problems from a long time in the areas of computer vision and image processing, signal processing and in various allied fields [11]. It is always seen that once the network is trained with suitable data sets it sets itself suitably to generalize and predict the output for any given input in that particular domain. Various structural variations of ANN are available in the literature making it more powerful for solving nonlinear classification problems. Multilayer perceptron (MLP) are capable to solve highly nonlinear problems in comparison to single perceptron, which are capable of solving simple linear Problems. FLANN belongs to the class of ANN, which is a flat network without any hidden layers [12, 13]. Back propagation training is much simpler with less computational complexity and faster convergence.

The proposed impulsive noise detector is shown in the Fig.3, which is a two-layered structure. The input to the network is coefficient of variance (CV) of the noisy image. This input is functionally expanded in the input layer with the trigonometric polynomial basis function given as

$$
\begin{aligned}
& 1, \sin (\pi C V), \sin (2 \pi C V), \cdots, \sin (N \pi C V), \\
& C V, \cos (\pi C V), \cos (2 \pi C V), \cdots, \cos (N \pi C V)
\end{aligned}
$$

In order to determine the error we compare the actual output of the network with the desired output. As per the error value we update the weight matrix between input and output layers using back propagation algorithm (BPA) [11]. We take an image say Pepper that is corrupted with impulsive noise of noise density between 0.01 and 0.30 in a step of 0.05 . Each corrupted image is subjected to the proposed filter varying the threshold from 0 to 1 in a step of 0.01 and the corresponding mean squared error (MSE) value is computed using the relation.

$$
M S E=\frac{1}{M \times N} \sum_{i=1}^{M} \sum_{j=1}^{N}\left(X_{i, j}-\hat{Y}_{i, j}\right)^{2}
$$

Where [NXM] is the size of the image $X i, j$ and $j Y i, j$ which represents the pixel values at $(\mathrm{i}, \mathrm{j})^{\text {th }}$ location of original image and restored image respectively. The minimum MSE and the corresponding threshold value called optimum threshold $p \theta_{\text {opinum }}$ ]are recorded. Since the MSE requires the original image for computation we cannot use it for threshold detection in real time applications. Here we use coefficient of variance (CV) which is the ratio of standard deviation(б) and mean $(\mu)$ which can be easily computed from the noisy image available and is used here for threshold prediction using a FLANN (Fig.3). 
For training the FLANN the input-output patterns $\left((C V)->\theta_{\text {optimun }}\right)$ For different noise levels are generated for different images like Peppers, Lisa and Lena etc. The training convergence for our neural network is shown in Fig 4.As per our observation MSE shows more acceptable results in comparison to FLANN-ATS [14] and other standard impulsive noise removal schemes. So we can say that our scheme is able to remove impulsive noise from images while reserving the edges and also provides good contrast enhancement . The image edge and details along with their contrast preservation is very much important for various applications where image details are required. This is (11) (10) (8) (9) achieved by applying selective median filtrations on the noisy pixels only. Since we are not applying median filtering to healthy pixels we are avoiding the blurring effect (loss of details) of median filter which is desired for better image enhancement. Now we can easily apply the unsharp masking for edge enhancement of the image without amplification of impulsive noise. The amplification factor ?depends upon the application where we use and the desired level of image details contrast needed by the user. For experimental purpose we have used ?as 0.5 .

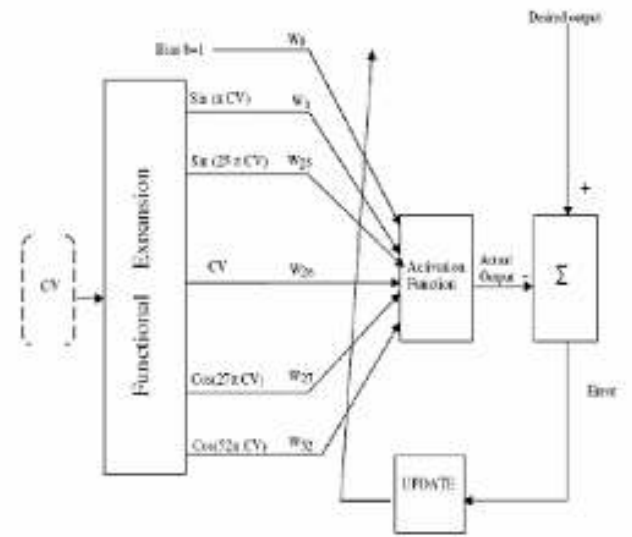

Fig. 3. FLANN stucture for adaptive threshold selection

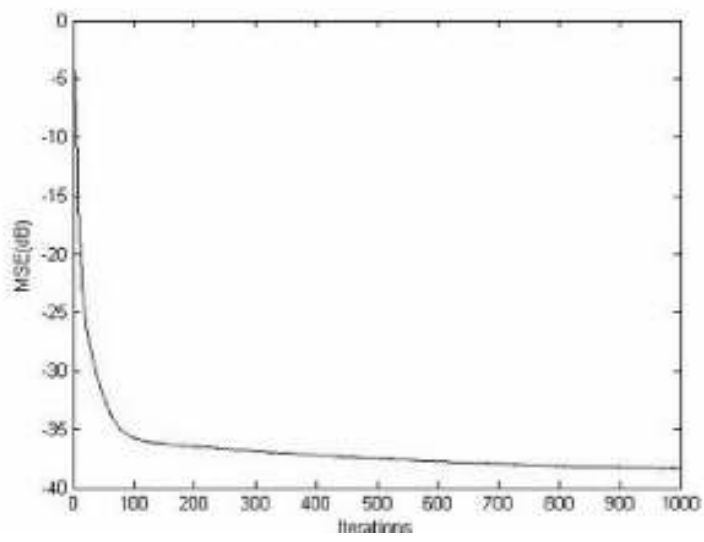

Fig. 4. Convergence characteristics of the network

\section{RESULTS AND DISCUSSION}

To demonstrate the performance of the proposed image enhancement scheme simulation results is being discussed under two heads: Impulsive noise removal and Image Contrast Enhancement.

\section{Impulsive Noise removal}

The superiority of the proposed impulse detection method is demonstrated here with the help of Peak Signal to Noise Ratio (PSNR).

PSNR $=10 \log _{10}\left(\frac{255^{2}}{M S E}\right) \mathrm{dB}$

PSNR is the metric, which is usually used for comparing superiority between image processing schemes. In order to confirm high performance of the proposed scheme, we demonstrate herewith some of the simulated results. Standard images like Lena, Lisa, Girl and Pepper are contaminated with noise ranging from 0.01 to 0.30 . It is then processed with various schemes like Progressive Switching Median(PSM)[15],Adaptive Center weighted Median Filter(ACWMF)[10],Two-Pass Median(two pass)[16] ,Switching Median(SWM(5X5))[3],Accurate Noise Detector(AND)[17], Two-Output Nonlinear Filter(2OUTPUT)[18],Median Rational hybrid FilterП?(MRHF2)[19], Detail Preserving Impulsive Noise removal(DPINR)[16],Impulse detection based on Pixel wise MAD(PWMAD)[17],FLANN based adaptive threshold selection for detection of impulsive noise in images(FLANN-ATS)[11] and proposed scheme. It is then processed with various schemes like Progressive Switching Median(PSM)[15],Adaptive Center weighted Median Filter(ACWMF) [7],Two-Pass Median(two pass)[4], Detail Preserving Impulsive Noise removal(DPINR)[20],Impulse detection based on Pixel wise MAD(PWMAD)[21],FLANN based adaptive threshold selection for detection of impulsive noise in images(FLANN-ATS)[14] and proposed scheme. Mean Squared Error (MSE) and Peak Signal to Noise Ratio (PSNR), as defined in (11) and (12) respectively are the two widely accepted measures used to compare the results of various noise removal schemes. Fig 5 shows the PSNR comparison between the proposed scheme and the existing schemes. Table 1 depict the comparative study of PSNR values for standard images (viz. Lena, Girl, Clown and Gatlin) contaminated with $15 \%$ of random valued impulsive noise. In terms of PSNR, in low noise condition there is not much difference among the simulated schemes but when the noise ratio increases the proposed scheme provides much superior results in comparison to other schemes. As per Table. 2. we can say our scheme 
gives better PSNR values in comparison to other impulsive noise removal schemes.

\section{Image contrast enhancement}

The performance of the proposed algorithm is compared with the help of three standard images lena, boat,pepper with the simple unsharp masking scheme as shown in Fig. 6. As an usual case of image enhancement there is no quantitative performance evaluation technique because of various reasons like: No ideal image can be used as reference. Any reasonable measure should be tuned to the human visual system. However perceptual quality evaluation is not a deterministic process. To be very specific we have no objective measure for comparing the results we go for subjective tests. It can be easily realized that since we are not amplifying the noise the quality is much better in (12) comparison to simple unsharp masking. Further we are using CLAHE for adaptive contrast enhancement. Since we are using CLAHE contrast enhancement is done in a controlled manner along with reduced chance for noise amplification.

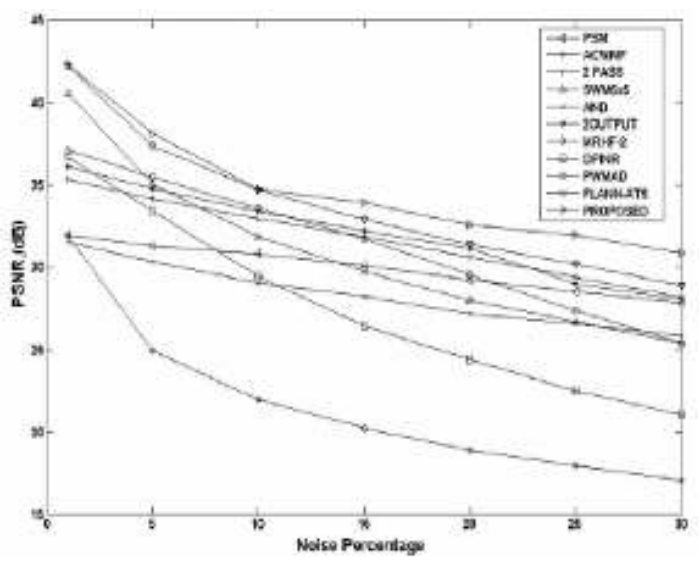

Fig. 5. PSNR for Lena image at various noise conditions

Table 2. Comparative results in PSNR (dB) of filtering Images

\begin{tabular}{|c|c|c|c|c|c|c|c|}
\hline blage: & ISSM & ACWMF & $294 \$ 5$ & DMINR & FWWAD & $\begin{array}{l}\text { FASS } \\
\text { ATs }\end{array}$ & PRCIPOSER \\
\hline Lens & 30.05 & 32.21 & 31.38 & 26.62 & 31.73 & 32.89 & 33.8901 \\
\hline Lisi & 29.09 & 31.78 & 31.34 & 27.36 & 30.50 & 30.84 & 31.6300 \\
\hline Bout & 26.78 & 23.87 & 29.33 & 25.87 & 29.25 & 29.93 & 30.4926 \\
\hline Clows: & 21.70 & 22.56 & 22.84 & 20.99 & 23.02 & 24.24 & 25.1320 \\
\hline
\end{tabular}
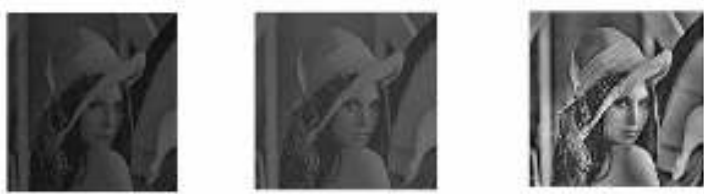

(a)Low contrastlena

(b)Linear unsharp masking

(C)Proposed
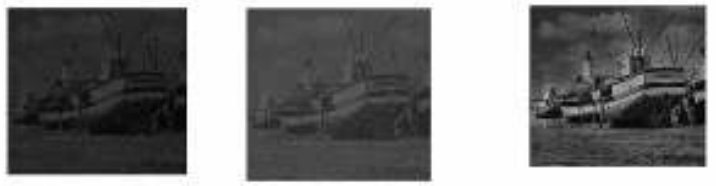

(a)Low contrast boat

(b)Linear unsharp masking

(c)Proposed
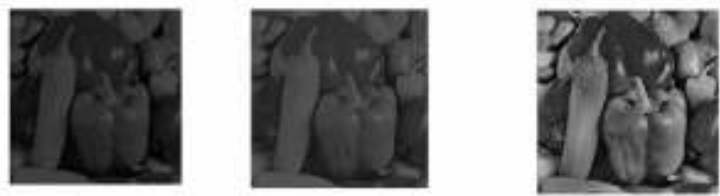

(A)Low contrast boat

(b)Linear unsharp masking

(c)Proposed

Fig. 6. Comparative Enhanced Images for Lena, Boat, and Pepper for (a) Low Contrast (b) Unsharp masking(c) Proposed

\section{CONCLUSION}

This paper has proposed a novel filtering scheme along with provision for contrast enhancement suppressing impulsive noise from contaminated images. The main idea is to use an alternate statistical parameter Coefficient of Variance $(\mathrm{CV})$ replacing mean and variance as used in FLANN-ATS [11] for determining threshold value without compromising the PSNR value. The filtration is performed selectively only on the detected noisy pixels segregating noisy pixels from noise-free pixels. Since avoiding unnecessary replacement of healthy pixels with its median value within its neighbors, we preserve the image details. We use unsharp masking followed by CLAHE for image contrast enhancement. Through exhaustive computer simulations on different standard images contaminated with varying noise densities it is observed that the proposed scheme exhibits superior performance over other schemes with provision for contrast enhancement and allowing easier hardware implementations.

\section{REFERENCES}

[1] Gonzalez and R. E. Woods, Digital Image Processing, 2nd Edition, Pearson Education.

[2] E.H. Hall, "Almost uniform distribution for computer image enhancement", IEEE trans Comput.23(2),1974,207-208 
[3] Y.-T. Kim Contrast Enhancement Using Brightness Preserving Bi-Histogram Equalization, IEEE Transactions on Consumer Electronics, Vol. 43. Feb 1997

[4] K.Wongsritong,K. Kittayaruasiriwat ,F.Cheevasuvit ,K.Dejhan and A.Somboonkaew, 1998, "Contrast enhancement using multipeak histogram equalization with brightness preserving" Circuits and Systems, IEEEAPCCAS

[5] Brownrigg DRK. The weighted median filter. Commun ACM 1984; 27:80718. [6] Chen T, Wu HR. Adaptive impulse detection using centerweighted median filters. IEEE Signal Process Lett 2001;8:13.

[7] HaykinS. Neural networks. 2nd ed., Englewood Cliffs, NJ Prentice-Hall; 1999. [8] Karel Zuiderveld, Contrast limited adaptive histogram equalization", Graphics gems IV, pages 474-485, 1994.

[9] J. S. Lee, "Digital image enhancement and noise filtering by using local statistics," IEEE Trans. Pattern Anal. Machine Intell. I. PAMI, no. 2, pp. 165168, 1980.

[10] P. M. Narendra and R. C. Fitch, "Real-time adaptive contrast enhancement,"IEEE Trans. Pattern Anal. Machine Intell., vol.PAMI, no. 3, pp. 655661, 1981.

[11] Chen T, Wu HR. Adaptive impulse detection using center weighted median filters. IEEE Signal Process Lett 2001;8:13.

[12] Patra JC, Pal RN, Chatterji BN, Panda G. Identification of nonlinear dynamic systems using functional link artificial neural networks. IEEE Trans Systems Man Cybernet 1999; 29: 25462.

[13] Patra JC, Panda G, Baliarsingh R. Artificial neural network based nonlinearity estimation of pressure sensors. IEEE Trans Instrum Meas 1994; 43:87481.
[14] FLANN-based adaptive threshold selection for detection of impulsive noise in images. B. Majhi and K. Sa AEU - International Journal of Electronics and Communications, Volume 61, Issue 7, Pages 478484

[15] Wang Z, Zhang D. Progressive switching median filter for the removal of impulse noise from highly corrupted images. IEEE Trans Circuits Systemsll: Analog Digital Signal Process 1999;46:7880.

[16] X. Xu and E. L. Miller, "Adaptive Two-Pass Median Filter to Remove Impulsive Noise", Proc.International Conference on Image Processing, 2002,vol. 1, pp. I-808I-811, 2225, Sep. 2002

[17] Kondo K, Haseyama M, Kitajima H. An accurate noise detector for image restoration. In: Proceedings of international conference on image processing, 2002. September 2002.132114.

[18] Russo F. Impulse noise cancellation in image data using a two-output nonlinear filters. Measurement 2004; 36:20513.

[19] Khriji L, Gabbouj M. Median-rational hybrid filters. In:Proceedings of international conference on image processing, 1998. October 1998. p. 8537.

[20] Alajlan N, Kamel M, Jernigan E. Detail preserving impulsive noise removal. Signal Process: Image Commun 2004; 19:9931003.

[21] Crnojevic V, Senk V, Trpovski Z. Advanced impulse detection based on pixel-wise MAD. IEEE Signal Process Lett 2004; 11:58992. 